\title{
Anatomical dissection of the calcaneofibular ligament of the ankle - a macroscopic and morphometric study
}

\author{
Assia Yabka ${ }^{1, A-F}{ }^{\oplus}$, Hamzaoui Bahia ${ }^{2, B} \odot$ \\ ${ }^{1}$ Université 1, Algiers, Algeria \\ 2 Saad Dahleb University, Algiers, Algeria \\ A - Research concept and design, B - Collection and/or assembly of data, C - Data analysis and interpretation, \\ $D$ - Writing the article, E-Critical revision of the article, F- Final approval of the article
}

Assia Yabka, Hamzaoui Bahia. Anatomical dissection of the calcaneofibular ligament of the ankle Macroscopic and morphometric study. J Pre-Clin Clin Res. 2021; 15(3): 121-125. doi: 10.26444/jpccr/140211

\begin{abstract}
Introduction and objective. Common to the talocrural and subtalar joints, the calcaneofibular ligament forms with the anterior bundle a mechanical coupling factor for ankle stabilization. Adhering to the deep surface of the fibular sheath, doubled in depth by the lateral talocalcaneal ligament, it is solicited in isolated varus and its lesion is involved in subtalar instability. The therapeutic strategy for chronic ankle instability is currently based on the anatomical reconstruction of the ligaments, particularly by arthroscopy, hence the objective of this study, which aims to highlight the great variability of the calcaneofibular ligament.

Materials and method. Our work is based on the dissection of 24 ankles. The number of beams, the dimensions at the neutral point and after power-up, the relationships with the surrounding anatomical structures as well as the points of insertion of the CFL are studied.

Results. The calcaneofibular ligament was monofascicular in $91.6 \%$ of cases and bifascicular in $8.4 \%$. Its average length in a neutral position was $26.85 \pm 3.46 \mathrm{~mm}$, and its width was $5.33 \pm 1.45 \mathrm{~mm}$. In dorsiflexion, the length was $27.10 \pm 0.05 \mathrm{~mm}$, and in forced inversion, it was $26.98 \pm 0.07 \mathrm{~mm}$. The distance between its fibular insertion and the apex of the lateral malleolus was $6.2 \pm 0.9 \mathrm{~mm}$.

Conclusions. Injuries of the calcaneofibular ligament are common in lateral ankle sprains, which can often get complicated by chronic instability. The macroscopic and morphometric knowledge of this ligament can allow a more accurate anatomical reconstruction and a more accurate choice of graft.
\end{abstract}

\section{Key words}

anatomical dissection, ankle ligaments, morphometric study, anatomical variations

\section{INTRODUCTION}

The stability of the ankle joint is passively ensured by the collateral ligaments, which are real means of contention. The calcaneofibular ligament $(\mathrm{CFL})$ represents the middle bundle of the lateral collateral ligament [1-2]. Common to both joints of the hindfoot, it plays an undeniable role in the lateral stability of the ankle. Some studies suggest that connective fibre interconnections between the three components of the lateral collateral ligament of the ankle can be observed from the medial surface of the ankle, which may have important implications for arthroscopic repair of the lateral ligament [3]. It is connected by collateral fibres to the distal bundle of the anterior talofibular ligament (ATFL) and forms with it a functional and anatomical entity called the fibulotalocalcaneal complex (LFTCL), the lesion of which leads to instability of the ankle [4]. The CFL, in the form of an elongated fibrous cord, oblique at the bottom, back and outside, extends from the lateral surface and the anterior edge of the fibular malleolus to the lateral surface of the calcaneus, behind the tubercle of the fibular tendons [2-5]. The CFL adheres to the deep surface of the fibular sheath, and can be

Address for correspondence: Assia Yabka, Université1 Alger. Algerie, Laboratoire d'Anatomie Générale université d'Alger, 16006, Alger, Algeria

E-mail:yabassia@gmail.com

Received: 08.01.2021; accepted: 14.07.2021; first published: 22.07.2021 doubled in depth by the lateral talocalcaneal ligament [6]. It lays the subtalar joint and supports the fibular tendons with which [7]. Solicited in isolated varus, its section intervenes in subtalar instability [8]. Its morphometric aspects have been reported in various cadaveric studies, its dimensions, on average, are $31 \pm 6 \mathrm{~mm}$ in length, and $4.4 \pm 3.1 \mathrm{~mm}$ in width, its angle with the sagittal plane $-51.11^{\circ}$. Its maximum elongation in extreme inversion and in dorsiflexion is $2.88 \%$ of its initial length [9-10]. The fibular insertion centre is 4.2 $\pm 1.8 \mathrm{~mm}$ from the apex of the lateral malleolus, and the calcaneal insertion centre is $18.4 \pm 2.5 \mathrm{~mm}$ from the apex of the fibular process of the calcaneus (radiological interest) [11]. The difference between the anatomical and arthroscopic centres of the calcaneofibular ligament insertions is $1.4 \pm$ $1.4 \mathrm{~mm}$ for the fibula and $4.1 \pm 2.3 \mathrm{~mm}$ for the calcaneus [11].

Like all ankle ligaments, the CFL consists of specialized connective tissue which has very interesting biomechanical properties that have the ability to adapt to the complex functions performed by this joint. Several cadaveric studies have revealed the presence of mechanoreceptors present throughout the ligament, in the periligamentous connective tissue and in partitions parallel to the ligament fibres, capable of detecting the speed of movement and alerting the nervous system [12]. The calcaneofibular ligament has a common origin with the anterior bundle in $33 \%$ of cases, and is replaced by the anterior talocalcaneal ligament in approximately $42 \%$ 
of cases [8]. CFL injury in lateral ankle sprains is rarely isolated, requiring a pure varus mechanism with dorsal hyperflexion. It is most often associated with anterior talofibular ligament injury (ATFL) [13]. The occurrence of chronic instability following a sprained ankle is probable, and can secondarily progress to post-traumatic osteoarthritis, and thus interfere with the quality of life of patients. The treatment of chronic ankle instability is mainly based on anatomical reconstruction of the lateral ankle ligaments, which must be isometric, respecting the anatomical features of the ligament [14].

The aim of this work is to show the anatomical, morphological and morphometric features of the CFL, the importance of which in the management of lateral ankle sprains, whether radiological or surgical is undeniable.

\section{MATERIALS AND METHOD}

The anatomical and morphometric study was carried out at the Laboratory of Anatomy, Organogenesis and Biomechanics of the Free University of Brussels in February 2016.

Twenty-four specimens were studied which had no known surgical history, divided as follows:

- Six fresh pieces of pelvic limbs, amputated at the union of the upper two-thirds of the leg and the lower third.

- Eighteen pieces of pelvic limbs embalmed and preserved in formaldehyde.

The material used was a basic dissection kit (forceps, scalpels, etc.), a millimetre ruler, scale protractor and a camera.

First, the incision lines of the instep area were identified using a dermographic pencil (Fig. 1).

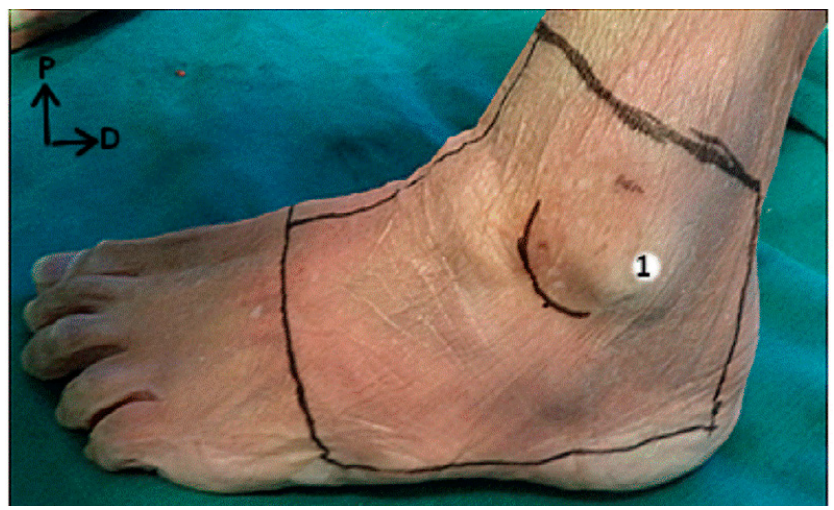

Figure 1. Side view of a left ankle showing anatomical landmarks (1) and incision lines. After incision of the skin, which was thin and supple and pulled the skin flaps, we demonstrated the superficial subcutaneous plan and the superficial vascular-neural elements

Dissection was continued to the lateral ligament complex of the talocrural joint.

The main points on which the study was based were: identifying the CFL beams, the precision of the insertions points, measuring its dimensions; length and width in neutral and after tensioning, comparing the values between right and left ankles, studying the relations with neighbouring structures, and finally searching for anatomical variability. The angle that this ligament makes with the longitudinal plane plays an important role in the stability of the ankle.
Care was taken in measuring it by keeping the ankle in a neutral position and using a protractor. Identifying its insertion points is important in anatomical reconstructions. The distance between its fibular insertion and the apex of the lateral malleolus was measured, and Excel software used to calculate mean values as well as standard deviations of the dimensions of the CFL.

\section{RESULTS}

Anatomical dissection of 24 ankles with an average age of $78 \pm 2.85$ revealed a calcaneofibular ligament in the form of a thin, elongated fibrous cord with the following anatomical features:

- in $91.6 \%$ (22/24 pieces) it was formed by a single beam (Fig. 2);

- in $100 \%$ (24/24 pieces) it fitted at the ventral edge of the lateral malleolus outside the insertion of the anterior talofibular ligament, and never on the apex (Fig. 2);

- in $100 \%$ (24/24 pieces) it ended behind and above the fibular tubercle (trochlear process) at the lateral face of the calcaneus (Fig. 2);

- the distance from its fibular insertion to the apex of the lateral malleolus, on average, was $6.2 \pm 0.9 \mathrm{~mm}$ (Fig. 2);

- its average neutral length was $26.85 \pm 3.46 \mathrm{~mm}$; average width $-5.33 \pm 1.45 \mathrm{~mm}$;

- the CFL belongs to the talocrural and subtalar joint. On all the parts studied, it was covered on its upper $2 / 3$ by fibular tendons and their sheath.

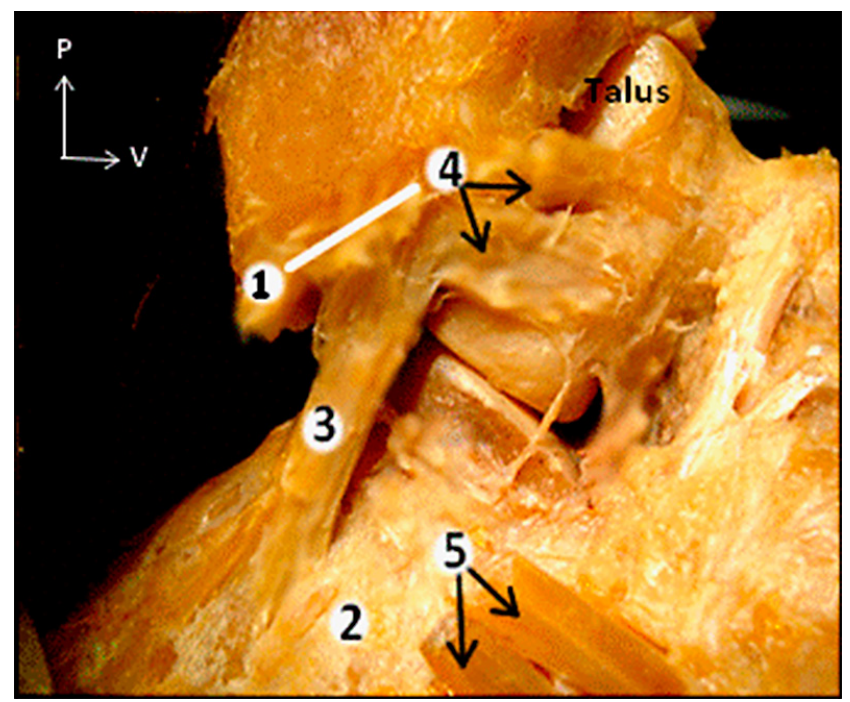

Figure 2. A single band forms the calcaneofibular ligament (3). The two-way arrow indicates the distance from its fibular insertion (4) to the apex of the lateral malleolus (1) [Brussels, 2016]. 2: calcaneus 5: sectioned fibular tendons

- in $8.4 \%$ (2/24 pieces), it was formed by 2 Y-shaped bundles (Fig. 3);

- a common origin between the ATFL and the CFL on the ventral edge of the fibular malleolus was found on one piece (Fig. 3).

- In the neutral position, the angle formed by the axis of the ligament with the sagittal plane averaged $53.70^{\circ}$ with a variance of $\left(35^{\circ}-58^{\circ}\right)$ (Fig. 4$)$. 


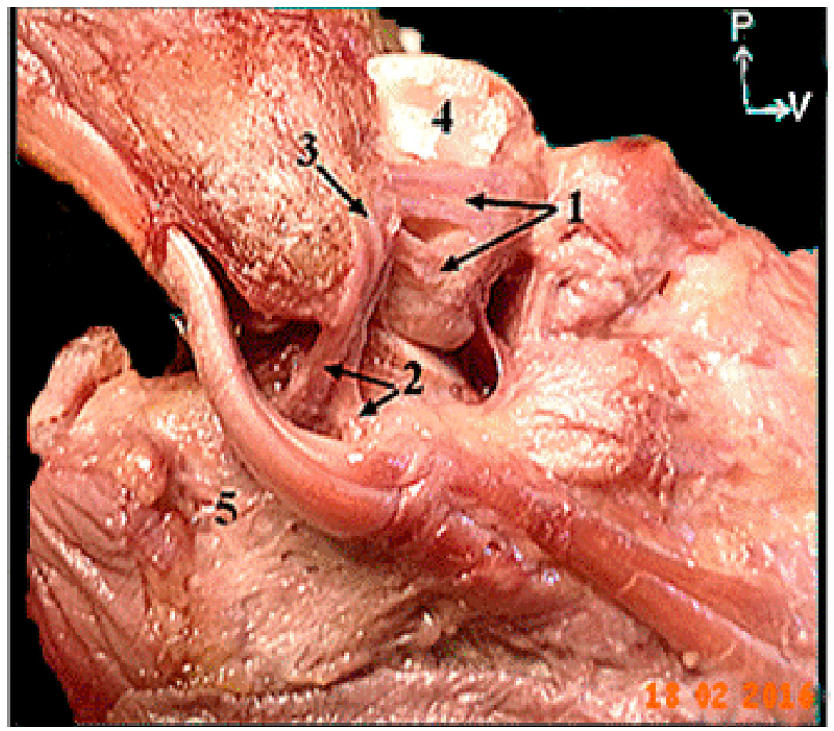

Figure 3. Lateral view of right ankle showing the 2 bundles of the anterior talofibular ligament (1), the 2 Y-shaped bundles of the calcaneofibular ligament (2), and their common origin on the ventral edge of the lateral malleolus (3). (4) Talus, (5) Lateral surface of the calcaneus [Brussels, 2016]

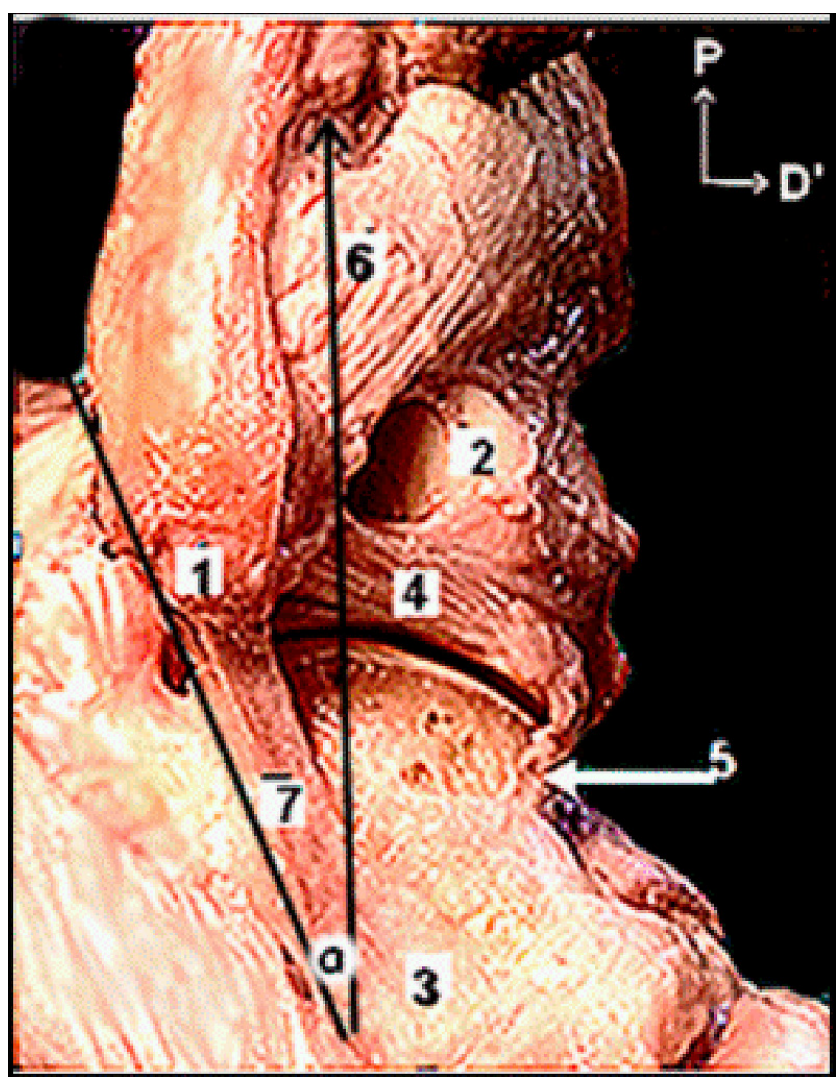

Figure 4. Lateral view of the ankle showing the calcaneofibular ligament and the angle it makes with the sagittal axis of the leg [Brussels, 2016]. 1 - Apex of the lateral malleolus; 2 - lateral face of the slope; 3 - Lateral aspect of the calcaneus; 4 - Posterior talofibular ligament; 5 - Talocalcaneal ligament; 6 - Posterior distal tibiofibular ligament; 7 - Calcaneofibular ligament a - Angle between the CFL and sagittal axis of the leg

During plantar flexion, the CFL is horizontal, and tight and vertical in dorsiflexion.

- In dorsiflexion, it undergoes an elongation of $0.25 \mathrm{~mm}$ $(27.10 \mathrm{~mm})$, thus an increase of $1.04 \%$ of its initial length (26.85 mm) (Fig. 5).

- Elongation in forced inversion - $0.13 \mathrm{~mm}(26.98 \mathrm{~mm})$.
Table 1. Comparison of LCF length and width parameters in $\mathrm{mm}$ between right and left ankles

\begin{tabular}{lcc}
\hline Ligament & Length & Width \\
\hline LCF & $26,85 \pm 3,46$ & $5,33 \pm 1,45$ \\
\hline Right side $(n=10)$ & $26,32 \pm 3,06$ & $5,05 \pm 1,02$ \\
\hline Left side $(n=14)$ & $25,87 \pm 2,66$ & $4,87 \pm 1,22$ \\
\hline Difference between right and left sides & $0.45 \pm 0.4$ & $0.18 \pm 0.2$ \\
\hline
\end{tabular}

Note that the right LCF exceeds the left LCF by a few $\mathrm{mm}$

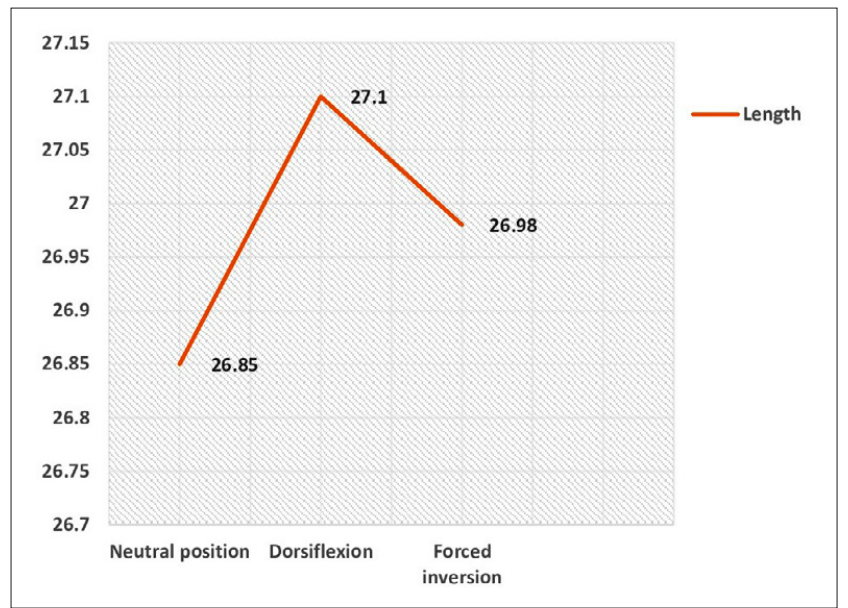

Figure 5. Variation in LCF length during power up. The lengthening of the LCF is greater in dorsiflexion than in forced inversion

\section{DISCUSSION}

The anatomy of the ankle ligaments is highly variable. Dissection on anatomical subjects, in addition to radiological imaging, allowed further knowledge to be gained.

The calcaneofibular ligament is involved in the stability of the talocrural and subtalar joints. Its involvement in the lateral ankle sprain is frequent, and is involved in the instability of the subtalar joint. Four distinct morphological forms of CFL are reported in the literature: a single bundle, a double Y-shaped bundle, a double V-shaped bundle, and a shape associated with the lateral talocalcaneal ligament.

In the study by Pereira BS et al., the shapes most commonly observed were single bundle and double bundle Y-shaped bundles in 21 (44.7\%) and 13 (27.7\%) ankles. The double V-bundle and double CFL bundle associated with the lateral talocalcaneal ligaments were less common [15]. Kitsoulis reported one beam in $72.2 \%$ of cases, 2 beams in $22.2 \%$, and 3 beams in $5.6 \%$ of the 72 pieces studied [7]. In the current study, the single radius shape occurred on 22 pieces $(92 \%)$ and the double $\mathrm{Y}$ shape on 2 pieces (8\%). The form associated with the talo-calcaneal ligament was absent.

The CFL shares some features with the distal bundle of the ATFL: 2 isometric ligaments with a common fibular insertion, connected by arciform fibres, and forming a functional and anatomical entity which has been named the lateral fibulotalocalcaneal ligament complex (LFTCL), the lesion of which can cause classical instability [4-16].

Kitsoulis, in his work on the morphology of the calcaneofibular ligament, reports a common insertion of the fibular with the anterior talofibular ligament in 33\% of cases, whereas Taser cited an anterior calcaneal termination 
in front of the fibular tubercle in $24(54 \%)$ of cases [7-17]. For the latter author [17], who worked on 44 ankles, the variation of the final insertion led to a modification of the angle of the CFL with the sagittal plane; his knowledge is interesting in the radiological diagnosis of ligamentous damage. According to the same authors, the orientation of the ligament also changes depending on the position of the talus, in varus or valgus, which could be a factor of instability and predispose to ankle sprains [7-17]. In the current study, a common insertion with the distal bundle of the ATFL was found in $4 \%$ of the cases. A dorsal calcaneal termination behind and above the tubercle of the fibulars - is constant on all the studied pieces, as described in the classical works.

The angle between the sagittal plane and the calcaneofibular ligament in the neutral position varies between $35^{\circ}-58^{\circ}$, with an average of $53.70^{\circ}$. These values are correlated with the measurements by Kitsoulis, which havean angle of $51.11^{\circ}$ and variance of $30^{\circ}-60^{\circ}$ ) and of Taser et al. of $51.14^{\circ}$ and variance of $32^{\circ}-60^{\circ}$ ). However, the measurements obtained in the current study are very different from the results of Burks and Morgan who have reported an angle of $133^{\circ}\left(113^{\circ}-150^{\circ}\right)$ [7-8-17]. Although the dimensions (length and width) are extremely variable from one author to another, the presented results are close to the data of the most recent study by Mahmut Ugurlu, where a difference occurs between the right and left sides, but is not significant, as indicated in the literature [18]. However, the predominance of straight values was also found in a study by Boulacel in 2008 on the biometry of the bone surfaces of the talocrural joint [19]. In fact, the values (length and width) of the talus are more important on the right, the degree of inclination formed by the edges; medial and lateral is higher on the right, and the fibular torsion is larger on the right, as is the depth of the tibial malleolus. Similarly, and according to Boulacel's [19] results, the index of the width of the trochlea is higher on the left. These results can be explained by the fact that the right pelvic limb predominates in dynamics, and therefore in mobility, while the left limb is more static and predominates in support and stability.

Identifying the insertion areas of this ligament the CFL is important in anatomical reconstructions. Clanton et al. gave the following values, the distance of its fibular insertion to the apex of the lateral malleolus $-5.3 \mathrm{~mm}(4.2-6.5 \mathrm{~mm})$ [20]. A cadaveric study confirmed the reliability of the lateral malleolus as a skin marker for the distal insertion of the central beam [21]. The precision of the fibular insertions of the anterior talofibular ligaments (ATFL) and calcaneofibularis (CFL), as well as their common origin with respect to the bony landmarks of the fibula, are necessary to determine the location of the fibular tunnel in reconstructive surgery [22]. The results obtained in the current study are relatively close and, on average, are $6.2 \pm 0.9 \mathrm{~mm}$. However, there is no consensus in the literature on a reproducible bone marker concerning the calcaneal insertion of the CFL.

The calcaneofibular ligament is stretched in forced inversion and dorsiflexion, and its length undergoes a maximum elongation of $2.88 \mathrm{~mm}$, on average, as observed by Kitsoulis, who also reports that women have a greater elongation than men, which could explain the ligamentous laxity among women, and which, according to Kitsoulis, would be due to hormonal factors [7]. In the current study, the elongation is very small, not exceeding $0.25 \mathrm{~mm}$, probably explained by the state of the dissected pieces that had lost their elasticity. Similarly, given the small number of dissected female ankles (4 pieces), ligamentous laxity could not be verified. However, it is proven that the lengthening of the calcaneofibular ligament is essential for the stabilization of the talocrural and subtalar joints during ankle sprains.

CFL injury is rarely isolated, requiring a pure varus mechanism with dorsal hyperflexion, and is most often associated with that of the ATFL. The problem is to locate the lesion of the calcaneofibular ligament [14].

\section{CONCLUSIONS}

The study of ankle ligaments is of particular interest in view of the development of surgical and exploratory techniques, not to mention the design of ankle prostheses.

1. Current trends in surgical treatment suggest the most anatomical reconstruction possible of the 2 anterior and middle bundles of the lateral collateral ligament.

2. Arthroscopic ligamentoplasty is increasingly used today, particularly in lateral ligament injuries of the ankle.

3. It appears from the results discussed that knowledge of the anatomical parameters of the ligaments is fundamental in the management of ankle sprains.

\section{REFERENCES}

1.Dufour M. Anatomie de l'appareil locomoteur T1. Editeur: ElsevierMasson. 3 Juin 2015. ISBN13: 9782294745027.546 p.

2. Yıldız S, Yalcın B. The anterior talofibular and calcaneofibular ligaments: an anatomic study. Surg Radiol Anat. 2013 Aug; 35(6): 511-6. doi: 10.1007/s00276-012-1071-3. Epub 2013 Jan 5. PMID: 23292089

3. Dalmau-Pastor M, Malagelada F, Calder J, et al. The lateral ankle ligaments are interconnected: the medial connecting fibres between the anterior talofibular, calcaneofibular and posterior talofibular ligaments. Knee Surg Sports Traumatol Arthrosc. 2020 Jan; 28(1): 34-39. doi: 10.1007/s00167-019-05794-8. Epub 2019 Nov 22. PMID: 31754730

4. Vega J, Malagelada F, Manzanares Céspedes MC, et al. The lateral fibulotalocalcaneal ligament complex: an ankle stabilizing isometric structure. Knee Surg Sports Traumatol Arthrosc. 2020 Jan; 28(1): 8-17. doi: 10.1007/s00167-018-5188-8. Epub 2018 Oct 29. PMID: 30374570

5. Kakegawa A, Mori Y, Tsuchiya A, et al. Independent Attachment of Lateral Ankle Ligaments: Anterior Talofibular and Calcaneofibular Ligaments - A Cadaveric Study. J Foot Ankle Surg. 2019 Jul; 58(4): 717722. doi: 10.1053/j.jfas.2018.12.009. Epub 2019 May 23. PMID: 31130481 6. Edama M, Kageyama I, Kikumoto T, et al. Morphological characteristics of the lateral talocalcaneal ligament: a large-scale anatomical study. Surg Radiol Anat. 2019 Jan; 41(1): 25-28. doi: 10.1007/s00276-018-2128-8 Epub 2018 Oct 30. PMID: 30377754

7. Kitsoulis P, Marini A, Pseftinakou A, et al. Morphological study of the calcaneofibular ligament in cadavers. Folia Morphol (Warsz). 2011 Aug; 70(3): 180-4. PMID: 21866529

8. Burks RT, Morgan J. Anatomy of the lateral ankle ligaments. Am J Sports Med. 1994 Jan-Feb; 22(1): 72-7. doi: 10.1177/036354659402200113 PMID: 8129114

9. Apoorva D, Lalitha C, Girish V Patil. "Morphometric Study of Calcanéofibular Ligament of Ankle". Journal of Evidence based Medicine and Healthcare; Volume 1, Issue 10, November 10, 2014 Page: 1268-1274. doi: $10.18410 / \mathrm{jebmh} / 2014 / 191$

10. Matsui K, Takao M, Tochigi Y, et al. Anatomy of anterior talofibular ligament and calcaneofibular ligament for minimally invasive surgery: a systematic review. Knee Surg Sports Traumatol Arthrosc. 2017 Jun; 25(6): 1892-1902. doi: 10.1007/s00167-016-4194-y. Epub 2016 Jun 13. PMID: 27295109

11. Thès A, Klouche S, Ferrand M, et al. Assessment of the feasibility of arthroscopic visualization of the lateral ligament of the ankle: a cadaveric study. Knee Surg Sports Traumatol Arthrosc. 2016 Apr; 24(4): 985-90. doi: 10.1007/s00167-015-3804-4. Epub 2015 Sep 26. PMID: 26410094 
12. Wu X, Song W, Zheng C, et al. Morphological study of mechanoreceptors in collateral ligaments of the ankle joint. J Orthop Surg Res. 10;92(2015). https://doi.org/10.1186/s13018-015-0215-7

13. Al-Mohrej OA, Al-Kenani NS. Acute ankle sprain: conservative or surgical approach? EFORT Open Rev. 2017 Mar 13; 1(2): 34-44. doi: 10.1302/2058-5241.1.000010. PMID: 28461926; PMCID: PMC5367574

14. Tourné Y, Mabit C. La cheville instable, de l'entorse récente à l'instabilité chronique. Elsevier Masson. 2015.

15. Pereira BS, van Dijk CN, Andrade R, et al. The calcaneofibular ligament has distinct anatomic morphological variants: an anatomical cadaveric study. Knee Surg Sports Traumatol Arthrosc. 2020 Jan; 28(1): 40-47. doi: 10.1007/s00167-019-05797-5. Epub 2019 Nov 27. PMID: 31776625

16. Szaro P, Ghali Gataa K, Polaczek M, et al. The double fascicular variations of the anterior talofibular ligament and the calcaneofibular ligament correlate with interconnections between lateral ankle structures revealed on magnetic resonance imaging. Sci Rep. 2020 Nov 27; 10(1) 20801. doi: 10.1038/s41598-020-77856-8. PMID: 33247207; PMCID: PMC7695848

17. Taser F, Shafiq Q, Ebraheim NA. Anatomy of lateral ankle ligaments and their relationship to bony landmarks. Surg Radiol Anat. 2006
Aug; 28(4): 391-7. doi: 10.1007/s00276-006-0112-1. Epub 2006 Apr 27. PMID: 16642279

18. Uğurlu M, Bozkurt M, Demirkale I, et al. Anatomy of the lateral complex of the ankle joint in relation to peroneal tendons, distal fibula and talus: a cadaveric study. Eklem Hastalik Cerrahisi. 2010 Dec; 21(3): 153-8. PMID: 21067497

19. Boulacel A. Étude morphologique et exploration de l'articulation talocrurale chez l'homme. Thèse soutenue le 13/10/2008. Université de Constantine (ed): 2007-2008.

20. Clanton TO, Campbell KJ, Wilson KJ, et al. Qualitative and Quantitative Anatomic Investigation of the Lateral Ankle Ligaments for Surgical Reconstruction Procedures. J Bone Joint Surg Am. 2014 Jun 18; 96(12): e98. doi: 10.2106/JBJS.M.00798. PMID: 24951749

21. Lopez R, Noailles T, Bouguennec N, et al. Validation anatomique d'un repérage cutané de l'insertion distale du LCF. Revue de chirurgie orthopédique et traumatologique. Volume 101, N85. Page. 33: doi: 10.1016/j.rcot.2015.09.391

22. Park JH, Kwon HW, Kim D, et al. The Location of the Fibular Tunnel for Anatomically Accurate Reconstruction of the Lateral Ankle Ligament: A Cadaveric Study. Biomed Res Int. 2021 Mar 18; 2021: 5575524. doi: 10.1155/2021/5575524. PMID: 33791364; PMCID: PMC7997753 